\title{
New Inequalities and Uncertainty Relations on Linear Canonical Transform Revisit
}

\author{
Xu Guanlei, ${ }^{1,2}$ Wang Xiaotong, ${ }^{1,2}$ and Xu Xiaogang ${ }^{2,3}$ \\ ${ }^{1}$ Department of Navigation, Dalian Naval Academy, Dalian 116018, China \\ ${ }^{2}$ Institute of Photoelectric Technology, Dalian of China, Dalian 116018, China \\ ${ }^{3}$ Department of Automatization, Naval Academy, Dalian 116018, China
}

Correspondence should be addressed to Xu Guanlei, xgl_86@163.com

Received 10 May 2009; Accepted 22 June 2009

Recommended by Ling Shao

\begin{abstract}
The uncertainty principle plays an important role in mathematics, physics, signal processing, and so on. Firstly, based on definition of the linear canonical transform (LCT) and the traditional Pitt's inequality, one novel Pitt's inequality in the LCT domains is obtained, which is connected with the LCT parameters $a$ and $b$. Then one novel logarithmic uncertainty principle is derived from this novel Pitt's inequality in the LCT domains, which is associated with parameters of the two LCTs. Secondly, from the relation between the original function and LCT, one entropic uncertainty principle and one Heisenberg's uncertainty principle in the LCT domains are derived, which are associated with the LCT parameters $a$ and $b$. The reason why the three lower bounds are only associated with LCT parameters $a$ and $b$ and independent of $c$ and $d$ is presented. The results show it is possible that the bounds tend to zeros.
\end{abstract}

Copyright ( $\odot 2009$ Xu Guanlei et al. This is an open access article distributed under the Creative Commons Attribution License, which permits unrestricted use, distribution, and reproduction in any medium, provided the original work is properly cited.

\section{Introduction}

The uncertainty principle is one elementary principle in signal processing [1-10] and physics [11-13]. For one given function $f(t) \in L^{1}(R) \cap L^{2}(R)$ (without loss of generalization, assuming $\|f(t)\|_{2}=1$ in the following of this paper) and its Fourier transform (FT) $F(u)$, the product has the lowest bound

$$
\begin{aligned}
\Delta t^{2} \cdot \Delta u_{c l}^{2}= & \int_{-\infty}^{+\infty}\left|\left(t-t_{0}\right) f(t)\right|^{2} d t \\
& \cdot \int_{-\infty}^{+\infty}\left|\left(u-u_{0}\right) F(u)\right|^{2} d u \geq \frac{1}{4},
\end{aligned}
$$

where $t_{0}=\int_{-\infty}^{+\infty} t|f(t)|^{2} d t, u_{0}=\int_{-\infty}^{+\infty} u|F(u)|^{2} d u, \Delta t^{2}$ is time spread, and $\Delta u_{c l}^{2}$ is frequency spread. Let $t_{0}=0$ (in our paper for given $f(t)$ we assume $\left.t_{0} \equiv 0\right)$ and $u_{0}=0$, and the essence of uncertainty principle will not change [1-10] . However (1) can be written as

$$
\begin{aligned}
\Delta t^{2} \cdot \Delta u_{c l}^{2}= & \int_{-\infty}^{+\infty}|t f(t)|^{2} d t \\
& \cdot \int_{-\infty}^{+\infty}|u F(u)|^{2} d u \geq \frac{1}{4} .
\end{aligned}
$$

In this paper we will give three uncertainty principles in the LCT domains: one logarithmic uncertainty principle based on Pitt's inequality [14-16]; one entropic uncertainty principle; one Heisenberg's uncertainty principle. Note that some of our results of this article are the extension and generality of our recent works [17-19], and it is likely that there is part of similarity in the process of derivation. However, the results of this paper and most of the derivation are different and novel. First, Heisenberg's uncertainty in the recent works, such as [18-22], has been involved. However, the results of $[18,22]$ only hold true for the real signals (not for complex signals). In addition, the result of [22] is only the first one of the three cases in [18]. In [19], Pitt's inequality and logarithmic uncertainty principle on LCT have not been involved. Moreover, the derivations here are different from that in [19]. On the other hand, the results in $[20,21]$ are only some special cases of those in $[18,19,22]$ for special parameters.

The LCT is taken as the generalization of the FRFT and the Fresnel transform and has been widely studied and applied [9, 23-27] up till now. As a generalization of the traditional FT and the FRFT, the LCT has some properties with its transformed parameter. For more details, 
see [9, 23-27] and so forth. We now briefly review its definition and some basic properties.

For given function $f(t) \in L^{1}(R) \cap L^{2}(R)$ and $\|f(t)\|_{2}=1$ (in this article supposing this always holds), its definition of the LCT [9] is

$$
\begin{aligned}
F_{(a, b, c, d)}(u) & =F_{(a, b, c, d)}(f(t))=\int_{-\infty}^{\infty} f(t) K_{a, b, c, d}(u, t) d t \\
& =\left\{\begin{array}{lc}
\sqrt{\frac{1}{i 2 \pi b}} \cdot e^{i d u^{2} / 2 b} \int_{-\infty}^{\infty} e^{-i u t / b} e^{i a t^{2} / 2 b} f(t) d t \\
b \neq 0, a d-b c=1 \\
\sqrt{d} \cdot e^{i c d u^{2} / 2} f(d u), & b=0,
\end{array}\right.
\end{aligned}
$$

where $a, b, c, d \in R$.

From the definition, it is easily found that

$$
F_{\left(a_{2}, b_{2}, c_{2}, d_{2}\right)}\left\{F_{\left(a_{1}, b_{1}, c_{1}, d_{1}\right)}(f(t))\right\}=F_{(a, b, c, d)}(f(t)),
$$

where $\left[\begin{array}{ll}a & b \\ c & d\end{array}\right]=\left[\begin{array}{ll}a_{2} & b_{2} \\ c_{2} & d_{2}\end{array}\right] \cdot\left[\begin{array}{ll}a_{1} & b_{1} \\ c_{1} & d_{1}\end{array}\right]$, and $i$ is complex unit.

For traditional FT which is a special case of $(a, b, c, d)=$ $(0,1,-1,0)$, we have

$$
\begin{aligned}
F_{(0,1,-1,0)}(u) & =F(u)=\sqrt{\frac{1}{2 \pi}} \int_{-\infty}^{\infty} f(t) e^{-i u t} d t, \\
f(t) & =\sqrt{\frac{1}{2 \pi}} \int_{-\infty}^{\infty} F(u) e^{i u t} d u .
\end{aligned}
$$

This paper is organized as follows. Section 2 yields the novel Pitt's inequality and the logarithmic uncertainty principle in the LCT domains. In Section 3 one novel entropic uncertainty principle is derived. In Section 4 Heisenberg's uncertainty principle is obtained. Finally, Section 5 concludes our paper.

\section{New Pitt's Inequality and Logarithmic Uncertainty Principle on LCT}

Inequalities [3, 14-16, 28, 29] are a basic tool in the study of Fourier analysis or information theory, and many important theorems or principles are derived from them. One of them is the Pitt's inequality by Beckner [14-16]:

$$
\int_{-\infty}^{\infty}|u|^{-\lambda}|F(u)|^{2} d u \leq M_{\lambda} \int_{-\infty}^{\infty}|t|^{\lambda}|f(t)|^{2} d t
$$

where $M_{\lambda}=[\Gamma((1-\lambda) / 4) / \Gamma((1+\lambda) / 4)]^{2}, 0 \leq \lambda<1, F(u)=$ $\sqrt{1 / 2 \pi} \int_{-\infty}^{\infty} f(t) e^{-i u t} d t$.

First we assume $a_{l}, b_{l}, c_{l}, d_{l} \in \mathbf{R}$ and $b_{l} \neq 0(l=1,2,3)$.

Set

$$
\begin{gathered}
G(u)=F_{\left(a_{1}, b_{1}, c_{1}, d_{1}\right)}(u) \exp \left(-i \frac{d_{3} u^{2}}{2 b_{3}}\right), \\
F_{\left(a_{1}, b_{1}, c_{1}, d_{1}\right)}(u)=F_{\left(a_{1}, b_{1}, c_{1}, d_{1}\right)}(f(t)) \\
g(t)=\sqrt{\frac{1}{2 \pi}} \int_{-\infty}^{\infty} G(u) e^{i u t} d u
\end{gathered}
$$

Noting the fact that $\left|F_{\left(a_{1}, b_{1}, c_{1}, d_{1}\right)}(u) \exp \left(-i d_{3} u^{2} / 2 b_{3}\right)\right|=$ $\left|F_{\left(a_{1}, b_{1}, c_{1}, d_{1}\right)}(u)\right|$ holds, we easily obtain

$$
\int_{-\infty}^{\infty}|u|^{-\lambda}|G(u)|^{2} d u=\int_{-\infty}^{\infty}|u|^{-\lambda}\left|F_{\left(a_{1}, b_{1}, c_{1}, d_{1}\right)}(u)\right|^{2} d u .
$$

From (6) and (8), we have

$$
\int_{-\infty}^{\infty}|u|^{-\lambda}\left|F_{\left(a_{1}, b_{1}, c_{1}, d_{1}\right)}(u)\right|^{2} d u \leq M_{\lambda} \int_{-\infty}^{\infty}|t|^{\lambda}|g(t)|^{2} d t .
$$

Noting $g(t)$, we have

$$
\begin{aligned}
\int_{-\infty}^{\infty}|t|^{\lambda}|g(t)|^{2} d t & =\int_{-\infty}^{\infty}\left|\frac{t}{b_{3}}\right|^{\lambda}\left|g\left(\frac{t}{b_{3}}\right)\right|^{2} d \frac{t}{b_{3}} \\
& =\frac{1}{\left|b_{3}\right|^{\lambda+1}} \int_{-\infty}^{\infty}|t|^{\lambda}\left|g\left(\frac{t}{b_{3}}\right)\right|^{2} d t
\end{aligned}
$$

Here from the definition of FT we have

$$
\left|g\left(\frac{t}{b_{3}}\right)\right|^{2}=\left|\sqrt{\frac{1}{2 \pi}} \int_{-\infty}^{\infty} G(u) e^{i u t / b_{3}} d u\right|^{2}
$$

Substituting $F_{\left(a_{1}, b_{1}, c_{1}, d_{1}\right)}(u) e^{-i d_{3} u^{2} / 2 b_{3}}$ for $G(u)$ in (11) and using definition (3), we get

$$
\begin{aligned}
& \left|g\left(\frac{t}{b_{3}}\right)\right|^{2} \\
& =\left|\sqrt{\frac{1}{2 \pi}} \int_{-\infty}^{\infty} F_{\left(a_{1}, b_{1}, c_{1}, d_{1}\right)}(u) e^{-i d_{3} u^{2} / 2 b_{3}} e^{i u t / b_{3}} d u\right|^{2} \\
& =\left|\frac{\sqrt{-1 / 2 i b_{3} \pi} \int_{-\infty}^{\infty} F_{\left(a_{1}, b_{1}, c_{1}, d_{1}\right)}(u) e^{-i d_{3} u^{2} / 2 b_{3}} e^{i u t / b_{3}} e^{-i a_{3} t^{2} / 2 b_{3}} d u}{\exp \left(-i a_{3} t^{2} / 2 b_{3}\right) \sqrt{-1 / i b_{3}}}\right|^{2} \\
& =\left|b_{3}\right|\left|\sqrt{\frac{-1}{2 i b_{3} \pi}} \int_{-\infty}^{\infty} F_{\left(a_{1}, b_{1}, c_{1}, d_{1}\right)}(u) e^{-i d_{3} u^{2} / 2 b_{3}} e^{i u t / b_{3}} e^{-i a_{3} t^{2} / 2 b_{3}} d u\right|^{2} \\
& =\left|b_{3}\right|\left|F_{\left(d_{3},-b_{3},-c_{3}, a_{3}\right)}\left(F_{\left(a_{1}, b_{1}, c_{1}, d_{1}\right)}\right)(t)\right|^{2} .
\end{aligned}
$$

Thus we obtain

$$
\begin{aligned}
& \int_{-\infty}^{\infty}|t|^{\lambda}|g(t)|^{2} d t \\
& \quad=\frac{1}{\left|b_{3}\right|^{\lambda}} \int_{-\infty}^{\infty}|t|^{\lambda}\left|F_{\left(d_{3},-b_{3},-c_{3}, a_{3}\right)}\left(F_{\left(a_{1}, b_{1}, c_{1}, d_{1}\right)}\right)(t)\right|^{2} d t .
\end{aligned}
$$

Set $t=v$, namely,

$$
\begin{aligned}
& \int_{-\infty}^{\infty}|u|^{-\lambda}\left|F_{\left(a_{1}, b_{1}, c_{1}, d_{1}\right)}(u)\right|^{2} d u \\
& \quad \leq \frac{M_{\lambda}}{\left|b_{3}\right|^{\lambda}} \int_{-\infty}^{\infty}|v|^{\lambda}\left|F_{\left(d_{3},-b_{3},-c_{3}, a_{3}\right)}\left(F_{\left(a_{1}, b_{1}, c_{1}, d_{1}\right)}\right)(v)\right|^{2} d v .
\end{aligned}
$$




$$
\begin{gathered}
\text { Let }\left[\begin{array}{ll}
a_{2} & b_{2} \\
c_{2} & d_{2}
\end{array}\right]=\left[\begin{array}{cc}
d_{3} & -b_{3} \\
-c_{3} & a_{3}
\end{array}\right] \cdot\left[\begin{array}{ll}
a_{1} & b_{1} \\
c_{1} & d_{1}
\end{array}\right] \text { have } \\
F_{\left(d_{3},-b_{3},-c_{3}, a_{3}\right)}\left(F_{\left(a_{1}, b_{1}, c_{1}, d_{1}\right)}(v)\right)=F_{\left(a_{2}, b_{2}, c_{2}, d_{2}\right)}(v) \\
b_{3}=-a_{1} b_{2}+a_{2} b_{1} .
\end{gathered}
$$

Comparing (14) with (15), we have

$$
\begin{aligned}
& \int_{-\infty}^{\infty}|u|^{-\lambda}\left|F_{\left(a_{1}, b_{1}, c_{1}, d_{1}\right)}(u)\right|^{2} d u \\
& \quad \leq \frac{M_{\lambda}}{\left|a_{1} b_{2}-a_{2} b_{1}\right|^{\lambda}} \int_{-\infty}^{\infty}|v|^{\lambda}\left|F_{\left(a_{2}, b_{2}, c_{2}, d_{2}\right)}(v)\right|^{2} d v .
\end{aligned}
$$

We can draw the conclusion that (16) is one extended Pitt's inequality in the LCT domains. It is easily found that this inequality is associated with LCT parameter $a, b$. Why do not the parameters $c, d$ have relation with the extended Pitt's inequality in the LCT domains? From definition (3) of the LCT, we find that the parameters $c, d$ only play the role of scaling and modulation. That the modulation has no effect on our (16) has been found from (8) and (12) directly. From the property $F_{(a, b, c, d)}(\sqrt{\rho} f(t / \rho))=F_{(a \rho, b / \rho, c \rho, d / \rho)}(f(t))$, we can easily find that scaling also has no effect on (16).

From definition (1) when $\left(a_{1}, b_{1}, c_{1}, d_{1}\right)=(0,1,-1,0)$ and $\left(a_{2}, b_{2}, c_{2}, d_{2}\right)=(1,0,0,1),(16)$ reduces to $(6)$. When $\left(a_{1}, b_{1}, c_{1}, d_{1}\right)=(1,0,0,1)$ and $\left(a_{2}, b_{2}, c_{2}, d_{2}\right)=(0,1,-1,0)$, (16) reads

$$
\int_{-\infty}^{\infty}|t|^{-\lambda}|f(t)|^{2} d t \leq M_{\lambda} \int_{-\infty}^{\infty}|u|^{\lambda}|F(u)|^{2} d u .
$$

Clearly, (17) is the other version of traditional Pitt's inequality. This is easily explained from the fact that $f(t)$ is also the FT of $F(u)$.

Particularly, if $\lambda=0$, from (16) we can get Parseval's equality [9] associated with the LCT:

$$
\int_{-\infty}^{\infty}\left|F_{\left(a_{1}, b_{1}, c_{1}, d_{1}\right)}(u)\right|^{2} d u=\int_{-\infty}^{\infty}\left|F_{\left(a_{2}, b_{2}, c_{2}, d_{2}\right)}(u)\right|^{2} d t .
$$

In the following, we will achieve one logarithmic uncertainty principle in the LCT domains.

Set $S(\lambda)=\left|a_{1} b_{2}-a_{2} b_{1}\right|^{\lambda} \int_{-\infty}^{\infty}|u|^{-\lambda}\left|F_{\left(a_{1}, b_{1}, c_{1}, d_{1}\right)}(u)\right|^{2} d u-$ $M_{\lambda} \int_{-\infty}^{\infty}|v|^{\lambda}\left|F_{\left(a_{2}, b_{2}, c_{2}, d_{2}\right)}(v)\right|^{2} d v$.

Then we have

$$
\begin{aligned}
S^{\prime}(\lambda)= & \left|a_{1} b_{2}-a_{2} b_{1}\right|^{\lambda} \ln \left(\left|a_{1} b_{2}-a_{2} b_{1}\right|\right) \\
& \times \int_{-\infty}^{\infty}|u|^{-\lambda}\left|F_{\left(a_{1}, b_{1}, c_{1}, d_{1}\right)}(u)\right|^{2} d u \\
& -\left|a_{1} b_{2}-a_{2} b_{1}\right|^{\lambda} \\
& \times \int_{-\infty}^{\infty}|u|^{-\lambda} \ln (|u|)\left|F_{\left(a_{1}, b_{1}, c_{1}, d_{1}\right)}(u)\right|^{2} d u \\
& -M_{\lambda} \int_{-\infty}^{\infty}|v|^{\lambda} \ln (|v|)\left|F_{\left(a_{2}, b_{2}, c_{2}, d_{2}\right)}(v)\right|^{2} d v \\
& -\left(M_{\lambda}\right)^{\prime} \int_{-\infty}^{\infty}|v|^{\lambda}\left|F_{\left(a_{2}, b_{2}, c_{2}, d_{2}\right)}(v)\right|^{2} d v,
\end{aligned}
$$

where $\left(M_{\lambda}\right)^{\prime}=\left(-(1 / 2) \Gamma((1-\lambda) / 4) \Gamma^{\prime}((1-\lambda) / 4) \Gamma^{2}((1+\lambda) / 4)-\right.$ $\left.(1 / 2) \Gamma((1+\lambda) / 4) \Gamma^{\prime}((1+\lambda) / 4) \Gamma^{2}((1-\lambda) / 4)\right) / \Gamma^{4}((1+\lambda) / 4)$.

Since $S(\lambda) \leq 0$ when $0 \leq \lambda<1$ and the fact $S(0)=0$ and $\int_{-\infty}^{\infty}\left|F_{\left(a_{1}, b_{1}, c_{1}, d_{1}\right)}(u)\right|^{2} d u=\int_{-\infty}^{\infty}\left|F_{\left(a_{2}, b_{2}, c_{2}, d_{2}\right)}(v)\right|^{2} d v=1$, we obtain the following inequality in mathematics $[11,30]$.

$$
S^{\prime}(0+) \leq 0 \text {. }
$$

Namely,

$$
\begin{aligned}
& \int_{-\infty}^{\infty} \ln |u|\left|F_{\left(a_{1}, b_{1}, c_{1}, d_{1}\right)}(u)\right|^{2} d u \\
& \quad+\int_{-\infty}^{\infty} \ln |v|\left|F_{\left(a_{2}, b_{2}, c_{2}, d_{2}\right)}(v)\right|^{2} d v \\
& \geq \ln \left|a_{1} b_{2}-a_{2} b_{1}\right|+\frac{\Gamma^{\prime}(1 / 4)}{\Gamma(1 / 4)} .
\end{aligned}
$$

From (21), we have

$$
\begin{aligned}
\int_{-\infty}^{\infty} & \ln |u|^{2}\left|F_{\left(a_{1}, b_{1}, c_{1}, d_{1}\right)}(u)\right|^{2} d u \\
& +\int_{-\infty}^{\infty} \ln |v|^{2}\left|F_{\left(a_{2}, b_{2}, c_{2}, d_{2}\right)}(v)\right|^{2} d v \\
\geq & \ln \left(\left|a_{1} b_{2}-a_{2} b_{1}\right|^{2}\right)+\frac{2 \Gamma^{\prime}(1 / 4)}{\Gamma(1 / 4)} .
\end{aligned}
$$

Clearly, the bound of the inequality (21) (or (22)) is connected with the LCT parameters $a$ and $b$ and independent of $c$ and $d$.

If

$$
\left[\begin{array}{ll}
a_{2} & b_{2} \\
c_{2} & d_{2}
\end{array}\right]=\left[\begin{array}{rr}
\vartheta & \vartheta-1 \\
1 & 1
\end{array}\right]
$$

where

$$
\begin{gathered}
\vartheta=\sqrt{-\frac{2 \Gamma^{\prime}(1 / 4)}{\Gamma(1 / 4)}} \\
{\left[\begin{array}{ll}
a_{1} & b_{1} \\
c_{1} & d_{1}
\end{array}\right]=\left[\begin{array}{rr}
0 & -1 \\
1 & 1
\end{array}\right],} \\
\ln \left(\left|a_{1} b_{2}-a_{2} b_{1}\right|^{2}\right)+\left(2 \Gamma^{\prime}(1 / 4)\right) /(\Gamma(1 / 4))=0 .
\end{gathered}
$$

It means that the bound of this inequality may be zero.

When $\left(a_{1}, b_{1}, c_{1}, d_{1}\right)=(\cos \alpha, \sin \alpha,-\sin \alpha, \cos \alpha)$ and $\left(a_{2}, b_{2}, c_{2}, d_{2}\right)=(\cos \beta, \sin \beta,-\sin \beta, \cos \beta),(22)$ reads

$$
\begin{gathered}
\int_{-\infty}^{\infty} \ln |u|^{2}\left|F_{\alpha}(u)\right|^{2} d u+\int_{-\infty}^{\infty} \ln |v|^{2}\left|F_{\beta}(v)\right|^{2} d v \\
\geq \ln \left(|\sin (\alpha-\beta)|^{2}\right)+\frac{2 \Gamma^{\prime}(1 / 4)}{\Gamma(1 / 4)} .
\end{gathered}
$$

In comparison with Heisenberg's uncertainty principle (28) in two fractional Fourier transform domains $[1,5,7]$ :

$$
\begin{gathered}
\int_{-\infty}^{\infty}|u|^{2}\left|F_{\alpha}(u)\right|^{2} d u \int_{-\infty}^{\infty}|v|^{2}\left|F_{\beta}(v)\right|^{2} d v \\
\geq \frac{|\sin (\alpha-\beta)|^{2}}{4}
\end{gathered}
$$


we find that there is one common term $|\sin (\alpha-\beta)|^{2}$ in (27) and (28). This tells us that in new transformed domains the new uncertainty principles have relations with the transform parameters. When $\left(a_{1}, b_{1}, c_{1}, d_{1}\right)=(1,0,0,1)$ and $\left(a_{2}, b_{2}, c_{2}, d_{2}\right)=(0,1,-1,0),(22)$ reads $\int_{-\infty}^{\infty} \ln |t||f(t)|^{2} d t+$ $\int_{-\infty}^{\infty} \ln |u||F(u)|^{2} d u \geq \Gamma^{\prime}(1 / 4) / \Gamma(1 / 4)$, which is the traditional logarithmic uncertainty principle by Beckner [16].

\section{Entropy and Entropic Uncertainty Principle on LCT}

The entropy is introduced by Shannon [31], and it has become one of the most important measures in information theory. The entropy has been widely used in many fields such as physics, communication, mathematics, signal analysis, and so forth.

The entropy is defined $[31,32]$ by

$$
E(\rho(x))=-\int_{-\infty}^{\infty} \rho(x) \ln \rho(x) d x
$$

where $\rho(x)$ is the probability density function of the variablex.

The entropic uncertainty principle plays one important role in signal processing and information theory. They are the extensions of traditional Heisenberg's uncertainty principle from time-frequency analysis to information theory and physical quantum. The traditional entropic uncertainty principle have been discussed in many papers such as [6, 10-13]. However, up till now there is no published paper covering the entropic uncertainty principle connected with the LCT. The traditional entropic uncertainty principle is described $[6,11-13]$ as

$$
\begin{gathered}
-\int_{-\infty}^{\infty}|f(t)|^{2} \ln |f(t)|^{2} d t-\int_{-\infty}^{\infty}|F(u)|^{2} \ln |F(u)|^{2} d u \\
\geq \ln (\pi e) .
\end{gathered}
$$

In the following, based on (30), the entropic uncertainty principle in two LCT domains is derived. $1,2,3)$.

First, similarly we assume $a_{l}, b_{l}, c_{l}, d_{l} \in \mathbf{R}$ and $b_{l} \neq 0(l=$

Set

$$
\begin{gathered}
G(u)=F_{\left(a_{1}, b_{1}, c_{1}, d_{1}\right)}(u) \exp \left(-i \frac{d_{3} u^{2}}{2 b_{3}}\right), \\
F_{\left(a_{1}, b_{1}, c_{1}, d_{1}\right)}(u)=F_{\left(a_{1}, b_{1}, c_{1}, d_{1}\right)}(f(t)), \\
g(t)=\sqrt{\frac{1}{2 \pi}} \int_{-\infty}^{\infty} G(u) e^{i u t} d u .
\end{gathered}
$$

Noting the fact that the equation

$$
\left|F_{\left(a_{1}, b_{1}, c_{1}, d_{1}\right)}(u) \exp \left(-i \frac{d_{3} u^{2}}{2 b_{3}}\right)\right|=\left|F_{\left(a_{1}, b_{1}, c_{1}, d_{1}\right)}(u)\right|
$$

holds, we easily get

$$
\begin{aligned}
& \int_{-\infty}^{\infty}|G(u)|^{2} \ln |G(u)|^{2} d u \\
& \quad=\int_{-\infty}^{\infty}\left|F_{\left(a_{1}, b_{1}, c_{1}, d_{1}\right)}(u)\right|^{2} \ln \left|F_{\left(a_{1}, b_{1}, c_{1}, d_{1}\right)}(u)\right|^{2} d u .
\end{aligned}
$$

From (30) and (33), we have

$$
\begin{aligned}
& -\int_{-\infty}^{\infty}|g(t)|^{2} \ln |g(t)|^{2} d t \\
& -\int_{-\infty}^{\infty}\left|F_{\left(a_{1}, b_{1}, c_{1}, d_{1}\right)}(u)\right|^{2} \ln \left|F_{\left(a_{1}, b_{1}, c_{1}, d_{1}\right)}(u)\right|^{2} d u \\
& \quad \geq \ln (\pi e) .
\end{aligned}
$$

Note the property of scaling:

$$
\begin{aligned}
& \int_{-\infty}^{\infty}|g(t)|^{2} \ln |g(t)|^{2} d t \\
& =\frac{1}{\left|b_{3}\right|} \int_{-\infty}^{\infty}\left|g\left(\frac{t}{b_{3}}\right)\right|^{2} \ln \left|g\left(\frac{t}{b_{3}}\right)\right|^{2} d t .
\end{aligned}
$$

Thinking about the definition of FT.

$$
\left|g\left(\frac{t}{b_{3}}\right)\right|^{2}=\left|\sqrt{\frac{1}{2 \pi}} \int_{-\infty}^{\infty} G(u) e^{i u t / b_{3}} d u\right|^{2}
$$

Similarly with (12), substituting $F_{\left(a_{1}, b_{1}, c_{1}, d_{1}\right)}(u) e^{-i d_{3} u^{2} / 2 b_{3}}$ for $G(u)$ in (36) and using definition (3), we get

$$
\begin{aligned}
\left|g\left(\frac{t}{b_{3}}\right)\right|^{2} & =\left|\sqrt{\frac{1}{2 \pi}} \int_{-\infty}^{\infty} F_{\left(a_{1}, b_{1}, c_{1}, d_{1}\right)}(u) e^{-i d_{3} u^{2} / 2 b_{3}} e^{i u t / b_{3}} d u\right|^{2} \\
& =\left|b_{3}\right|\left|F_{\left(d_{3},-b_{3},-c_{3}, a_{3}\right)}\left(F_{\left(a_{1}, b_{1}, c_{1}, d_{1}\right)}\right)(t)\right|^{2} .
\end{aligned}
$$

Thus we obtain

$$
\begin{aligned}
& \int_{-\infty}^{\infty}|g(t)|^{2} \ln |g(t)|^{2} d t \\
& =\frac{1}{\left|b_{3}\right|} \int_{-\infty}^{\infty}\left(\left|b_{3}\right|\left|F_{\left(d_{3},-b_{3},-c_{3}, a_{3}\right)}\left(F_{\left(a_{1}, b_{1}, c_{1}, d_{1}\right)}\right)(t)\right|^{2}\right) \\
& \quad \times \ln \left(\left|b_{3}\right|\left|F_{\left(d_{3},-b_{3},-c_{3}, a_{3}\right)}\left(F_{\left(a_{1}, b_{1}, c_{1}, d_{1}\right)}\right)(t)\right|^{2}\right) d t .
\end{aligned}
$$

Set $t=v$, then

$$
\begin{aligned}
& -\frac{1}{\left|b_{3}\right|} \int_{-\infty}^{\infty}\left(\left|b_{3}\right|\left|F_{\left(d_{3},-b_{3},-c_{3}, a_{3}\right)}\left(F_{\left(a_{1}, b_{1}, c_{1}, d_{1}\right)}\right)(v)\right|^{2}\right) \\
& \times \ln \left(\left|b_{3}\right|\left|F_{\left(d_{3},-b_{3},-c_{3}, a_{3}\right)}\left(F_{\left(a_{1}, b_{1}, c_{1}, d_{1}\right)}\right)(v)\right|^{2}\right) d v \\
& -\int_{-\infty}^{\infty}\left|F_{\left(a_{1}, b_{1}, c_{1}, d_{1}\right)}(u)\right|^{2} \ln \left|F_{\left(a_{1}, b_{1}, c_{1}, d_{1}\right)}(u)\right|^{2} d u \geq \ln (\pi e) .
\end{aligned}
$$


Set $\left[\begin{array}{ll}a_{2} & b_{2} \\ c_{2} & d_{2}\end{array}\right]=\left[\begin{array}{cc}d_{3} & -b_{3} \\ -c_{3} & a_{3}\end{array}\right] \cdot\left[\begin{array}{ll}a_{1} & b_{1} \\ c_{1} & d_{1}\end{array}\right]$, then we have

$$
\begin{gathered}
F_{\left(d_{3},-b_{3},-c_{3}, a_{3}\right)}\left(F_{\left(a_{1}, b_{1}, c_{1}, d_{1}\right)}(v)\right)=F_{\left(a_{2}, b_{2}, c_{2}, d_{2}\right)}(v), \\
b_{3}=-a_{1} b_{2}+a_{2} b_{1} .
\end{gathered}
$$

Comparing (39) with (40), we have

$$
\begin{aligned}
& -\int_{-\infty}^{\infty}\left|F_{\left(a_{2}, b_{2}, c_{2}, d_{2}\right)}(v)\right|^{2} \ln \left(\left|F_{\left(a_{2}, b_{2}, c_{2}, d_{2}\right)}(v)\right|^{2}\right) d v \\
& -\int_{-\infty}^{\infty}\left|F_{\left(a_{1}, b_{1}, c_{1}, d_{1}\right)}(u)\right|^{2} \ln \left|F_{\left(a_{1}, b_{1}, c_{1}, d_{1}\right)}(u)\right|^{2} d u \\
& \quad \geq \ln \left(\pi e\left|a_{1} b_{2}-a_{2} b_{1}\right|\right) .
\end{aligned}
$$

Namely, $E\left(\left|F_{\left(a_{1}, b_{1}, c_{1}, d_{1}\right)}(u)\right|^{2}\right)+E\left(\left|F_{\left(a_{2}, b_{2}, c_{2}, d_{2}\right)}(v)\right|^{2}\right) \geq$ $\ln \left(\pi e\left|a_{1} b_{2}-a_{2} b_{1}\right|\right)$.

Clearly, the entropic uncertainty principle in the LCT domains (see (41)) is connected with the LCT parameters $a$ and $b$ and independent of $c$ and $d$. Why do not the parameters $c, d$ have relation with the entropic uncertainty principle in the LCT domains? From definition (3) of the LCT, we find that the parameters $c, d$ only play the role of scaling and modulation. That the modulation has no effect on our inequality (41) has been found from (33) and (37) directly. From the property $F_{(a, b, c, d)}(\sqrt{\rho} f(t / \rho))=$ $F_{(a \rho, b / \rho, c \rho, d / \rho)}(f(t))$, we can easily find that scaling also has no effect on (41) as well as above shown. Similarly, if $\left[\begin{array}{ll}a_{1} & b_{1} \\ c_{1} & d_{1}\end{array}\right]=$ $\left[\begin{array}{cc}0 & -1 \\ 1 & 1\end{array}\right]$ and $\left[\begin{array}{ll}a_{2} & b_{2} \\ c_{2} & d_{2}\end{array}\right]=\left[\begin{array}{cc}1 / \pi e & 1 / \pi e-1 \\ 1 & 1\end{array}\right], \ln \left(\pi e\left|a_{1} b_{2}-a_{2} b_{1}\right|\right)=$ 0 . It means that the bound of this entropic uncertainty principle may be zero.

When $\left(a_{1}, b_{1}, c_{1}, d_{1}\right)=(\cos \alpha, \sin \alpha,-\sin \alpha, \cos \alpha)$ and $\left(a_{2}, b_{2}, c_{2}, d_{2}\right)=(\cos \beta, \sin \beta,-\sin \beta, \cos \beta),(41)$ reads

$$
\begin{aligned}
& -\int_{-\infty}^{\infty}\left|F_{\alpha}(v)\right|^{2} \ln \left(\left|F_{\alpha}(v)\right|^{2}\right) d v \\
& -\int_{-\infty}^{\infty}\left|F_{\beta}(u)\right|^{2} \ln \left|F_{\beta}(u)\right|^{2} d u \\
& \geq \ln (\pi e|\sin (\alpha-\beta)|) .
\end{aligned}
$$

Clearly, (42) is the entropic uncertainty principle in the fractional Fourier transform domains.

When $\left(a_{1}, b_{1}, c_{1}, d_{1}\right)=(1,0,0,1)$ and $\left(a_{2}, b_{2}, c_{2}, d_{2}\right)=$ $(0,1,-1,0),(41)$ reduces to the traditional case $(30)$.

\section{Heisenberg's Uncertainty Principle on LCT}

As (1), (2) showing, Heisenberg's uncertainty principle mainly discusses the product of time spread and frequency spread. In the same manner as Section 3, in this section, Heisenberg's uncertainty principle in the LCT domains is derived. Without loss of generality, assuming the mean values of the variables are zeros, namely,

$$
\int_{-\infty}^{+\infty}|t|^{2}|f(t)|^{2} d t \cdot \int_{-\infty}^{+\infty}|u|^{2}|F(u)|^{2} d u \geq \frac{1}{4} .
$$

First, similarly we assume $a_{l}, b_{l}, c_{l}, d_{l} \in \mathbf{R}$ and $b_{l} \neq 0(l=$ $1,2,3)$.

Set

$$
\begin{gathered}
G(u)=F_{\left(a_{1}, b_{1}, c_{1}, d_{1}\right)}(u) \exp \left(-i \frac{d_{3} u^{2}}{2 b_{3}}\right), \\
F_{\left(a_{1}, b_{1}, c_{1}, d_{1}\right)}(u)=F_{\left(a_{1}, b_{1}, c_{1}, d_{1}\right)}(f(t)), \\
g(t)=\sqrt{\frac{1}{2 \pi}} \int_{-\infty}^{\infty} G(u) e^{i u t} d u .
\end{gathered}
$$

Noting the fact that the equation

$$
\left|F_{\left(a_{1}, b_{1}, c_{1}, d_{1}\right)}(u) \exp \left(-i \frac{d_{3} u^{2}}{2 b_{3}}\right)\right|=\left|F_{\left(a_{1}, b_{1}, c_{1}, d_{1}\right)}(u)\right|
$$

holds, we easily obtain

$$
\int_{-\infty}^{+\infty}|u|^{2}|G(u)|^{2} d u=\int_{-\infty}^{+\infty}|u|^{2}\left|F_{\left(a_{1}, b_{1}, c_{1}, d_{1}\right)}(u)\right|^{2} d u .
$$

From (43) and (46), we have

$$
\int_{-\infty}^{+\infty}|t|^{2}|g(t)|^{2} d t \cdot \int_{-\infty}^{+\infty}|u|^{2}\left|F_{\left(a_{1}, b_{1}, c_{1}, d_{1}\right)}(u)\right|^{2} d u \geq \frac{1}{4}
$$

Through variable's scaling, we have

$$
\begin{aligned}
\int_{-\infty}^{+\infty}|t|^{2}|g(t)|^{2} d t & =\int_{-\infty}^{+\infty}\left|\frac{t}{b_{3}}\right|^{2}\left|g\left(\frac{t}{b_{3}}\right)\right|^{2} d\left(\frac{t}{b_{3}}\right) \\
& =\frac{1}{\left|b_{3}\right|^{3}} \int_{-\infty}^{+\infty}|t|^{2}\left|g\left(\frac{t}{b_{3}}\right)\right|^{2} d t .
\end{aligned}
$$

Meanwhile noting

$$
\left|g\left(\frac{t}{b_{3}}\right)\right|^{2}=\left|\sqrt{\frac{1}{2 \pi}} \int_{-\infty}^{\infty} G(u) e^{i u t / b_{3}} d u\right|^{2}
$$

Similarly with (12), substituting $F_{\left(a_{1}, b_{1}, c_{1}, d_{1}\right)}(u) e^{-i d_{3} u^{2} / 2 b_{3}}$ for $G(u)$ in (49) and using definition (3), we get

$$
\begin{aligned}
\left|g\left(\frac{t}{b_{3}}\right)\right|^{2} & =\left|\sqrt{\frac{1}{2 \pi}} \int_{-\infty}^{\infty} F_{\left(a_{1}, b_{1}, c_{1}, d_{1}\right)}(u) e^{-i d_{3} u^{2} / 2 b_{3}} e^{i u t / b_{3}} d u\right|^{2} \\
& =\left|b_{3}\right|\left|F_{\left(d_{3},-b_{3},-c_{3}, a_{3}\right)}\left(F_{\left(a_{1}, b_{1}, c_{1}, d_{1}\right)}\right)(t)\right|^{2} .
\end{aligned}
$$

Thus we obtain

$$
\begin{aligned}
& \int_{-\infty}^{+\infty}|t|^{2}|g(t)|^{2} d t \\
& =\frac{1}{\left|b_{3}\right|^{2}} \int_{-\infty}^{+\infty}|t|^{2}\left(\left|F_{\left(d_{3},-b_{3},-c_{3}, a_{3}\right)}\left(F_{\left(a_{1}, b_{1}, c_{1}, d_{1}\right)}\right)(t)\right|^{2}\right) d t
\end{aligned}
$$


Set $t=v$, then get

$$
\begin{gathered}
\frac{1}{\left|b_{3}\right|^{2}} \int_{-\infty}^{+\infty}|v|^{2}\left(\left|F_{\left(d_{3},-b_{3},-c_{3}, a_{3}\right)}\left(F_{\left(a_{1}, b_{1}, c_{1}, d_{1}\right)}\right)(v)\right|^{2}\right) d v \\
\cdot \int_{-\infty}^{+\infty}|u|^{2}\left|F_{\left(a_{1}, b_{1}, c_{1}, d_{1}\right)}(u)\right|^{2} d u \geq \frac{1}{4} .
\end{gathered}
$$

From (15) and (40), compared (51) with (52), we have

$$
\begin{aligned}
& \int_{-\infty}^{+\infty}|u|^{2}\left|F_{\left(a_{1}, b_{1}, c_{1}, d_{1}\right)}(u)\right|^{2} d u \\
& \cdot \int_{-\infty}^{+\infty}|v|^{2}\left(F_{\left(a_{2}, b_{2}, c_{2}, d_{2}\right)}(v)^{2}\right) d v \\
& \geq \frac{\left|a_{1} b_{2}-a_{2} b_{1}\right|^{2}}{4} .
\end{aligned}
$$

Clearly, Heisenberg's uncertainty principle in the LCT domains (see (53)) is only connected with the LCT parameters $a$ and $b$ and independent of $c$ and $d$. Why do not the parameters $c, d$ have relation with the entropic uncertainty principle in the LCT domains? The reasons are the same as those in Sections 2 and 3. When $a_{1} b_{2}-a_{2} b_{1} \rightarrow 0$, the bound of (53) tends to be zero.

When $\left(a_{1}, b_{1}, c_{1}, d_{1}\right)=(\cos \alpha, \sin \alpha,-\sin \alpha, \cos \alpha)$ and $\left(a_{2}, b_{2}, c_{2}, d_{2}\right)=(\cos \beta, \sin \beta,-\sin \beta, \cos \beta),(53)$ reads

$$
\begin{gathered}
\int_{-\infty}^{+\infty}|u|^{2}\left|F_{\alpha}(u)\right|^{2} d u \cdot \int_{-\infty}^{+\infty}|v|^{2}\left(F_{\beta}(v)^{2}\right) d v \\
\quad \geq \frac{|\sin (\alpha-\beta)|^{2}}{4} .
\end{gathered}
$$

However (54) is the Heisenberg's uncertainty principle in the fractional Fourier transform domains $[1,5,7,17]$. When $\left(a_{1}, b_{1}, c_{1}, d_{1}\right)=(1,0,0,1)$ and $\left(a_{2}, b_{2}, c_{2}, d_{2}\right)=(0,1,-1,0)$, (53) reduces to the traditional case $(43)$.

\section{Conclusions}

Three uncertainty principles associated with the LCT are presented in this paper. Firstly, from definition of LCT and the traditional Pitt's inequality, one novel Pitt's inequality in the LCT domains is obtained, which is connected with the LCT parameters $a$ and $b$ and independent of the LCT parameters $c$ and $d$. Then one novel logarithmic uncertainty principle is derived from this novel Pitt's inequality in two LCT domains. Secondly, based on the relation between one original function and LCT, the entropic uncertainty principle in two LCT domains is proposed. Thirdly, from the relation between one original function and its LCT, Heisenberg's uncertainty principle in two LCT domains is obtained. Note that the three lower bounds are only associated with LCT parameters $a$ and $b$ and independent of $c$ and $d$. In addition, the reasons are given. Moreover, one clear observation is that our three uncertainty principles hold for both real and complex signals. Our future work includes finding out how these cases can be generalized to discrete and multidimensional signals.

\section{Acknowledgment}

This work was partly supported by the NNSF of China and the Kaifang Foundation of Zhejiang University.

\section{References}

[1] G. Hardy, J. E. Littlewood, and G. Pólya, Inequalities, Press of University of Cambridge, Cambridge, UK, 2nd edition, 1951.

[2] K. K Selig, "Uncertainty principles revisited," Tech. Rep., Technische Universitat Munchen, 2001, http://www.emis.de/ journals/ETNA/vol.14.2002/pp165-177.dir/abstr165-177.ps.

[3] S. Shinde and V. M. Gadre, "An uncertainty principle for real signals in the fractional fourier transform domain," IEEE Transactions on Signal Processing, vol. 49, no. 11, pp. 25452548, 2001.

[4] D. Mustard, "Uncertainty principle invariant under fractional fourier transform," Journal of the Australian Mathematical Society, Series B, vol. 33, pp. 180-191, 1991.

[5] H. M. Ozaktas and O. Aytur, "Fractional fourier domains," Journal of Signal Processing, vol. 46, pp. 119-124, 1995.

[6] G. B. Folland and A. Sitaram, "The uncertainty principle: a mathematical survey," Journal of Fourier Analysis and Applications, vol. 3, no. 3, pp. 207-238, 1997.

[7] O. Aytur and H. M. Ozaktas, "Non-orthogonal domains in phase space of quantum optics and their relation to fractional fourier transforms," Optics Communications, vol. 120, no. 3-4, pp. 166-170, 1995.

[8] X. D. Zhang, Modern Signal Processing, Tsinghua University Press, Beingjing, China, 2nd edition, 2002.

[9] R. Tao, L. Qi, and Y. Wang, Theory and Application of the Fractional Fourier Transform, Tsinghua university Press, Beingjing, China, 2004.

[10] P. J. Loughlin and L. Cohen, "The uncertainty principle: global, local, or both?” IEEE Signal Processing SocietyTransactions on Signal Processing, vol. 52, no. 5, pp. 1218-1227, 2004.

[11] H. Maassen, "A discrete entropic uncertainty relation," in Quantum Probability and Applications, vol. 5, pp. 263-266, Springer, New York, NY, USA, 1988.

[12] I. I. Hirschman Jr., "A note on entropy," American Journal of Mathematics, vol. 79, no. 1, pp. 152-156, 1957.

[13] I. B. Birula, "Entropic uncertainty relations in quantum mechanics," in Quantum Probability and Applications II, L. Accardi and W. von Waldenfels, Eds., vol. 1136 of Lecture Notes in Mathematics, pp. 90-103, Springer, Berlin, Germany, 1985.

[14] W. Beckner, "Pitt's inequality and the uncertainty principle," Proceedings of the American Mathematical Society, vol. 123, no. 6, pp. 1897-1905, 1995.

[15] W. Beckner, "Pitt's inequality with sharp error estimates," accepted, http://arxiv.org/abs/math/0701939.

[16] W. Beckner, "Pitt's inequality with sharp convolution estimates," http://arxiv.org/abs/math/0701939.

[17] X. Guanlei, W. Xiaotong, and X. Xiaogang, "The logarithmic, heisenberg's and short-time uncertainty principles associated with fractional fourier transform," Signal Processing, vol. 89, no. 3, pp. 339-343, 2009.

[18] X. Guanlei, W. Xiaotong, and X. Xiaogang, “Three uncertainty relations for real signals associated with linear canonical transform," IET Signal Processing, vol. 3, no. 1, pp. 85-92, 2009. 
[19] X. Guanlei, W. Xiaotong, and X. Xiaogang, "Uncertainty inequalities for linear canonical transform," IET Signal Processing, vol. 3, no. 5, pp. 392-402, 2009.

[20] A. Stern, "Sampling of compact signals in offset linear canonical transform domains," Signal, Image and Video Processing, vol. 1, no. 4, pp. 359-367, 2007.

[21] A. Stern, "Uncertainty principles in linear canonical transform domains and some of their implications in optics," Journal of the Optical Society of America A, vol. 25, no. 3, pp. 647-652, 2008.

[22] K. K. Sharma and S. D. Joshi, "Uncertainty principle for real signals in the linear canonical transform domains," IEEE Transactions on Signal Processing, vol. 56, no. 7, pp. 2677-2683, 2008.

[23] M. Moshinsky and C. Quesne, "Linear canonical transformations and their unitary representation," Journal of Mathematical Physics, vol. 12, no. 8, pp. 1772-1783, 1971.

[24] X. Guanlei, W. Xiaotong, and X. Xiaogang, "Fractional quaternion fourier transform, convolution and correlation," Signal Processing, vol. 88, no. 10, pp. 2511-2517, 2008.

[25] B. Barshan, M. A. Kutay, and H. M. Ozaktas, "Optimal filtering with linear canonical transformations," Optics Communications, vol. 135, no. 1-3, pp. 32-36, 1997.

[26] S.-C. Pei and J.-J. Ding, "Relations between fractional operations and time-frequency distributions, and their applications," IEEE Transactions on Signal Processing, vol. 49, no. 8, pp. 1638-1655, 2001.

[27] S.-C. Pei and J.-J. Ding, "Two-dimensional affine generalized fractional fourier transform," IEEE Transactions on Signal Processing, vol. 49, no. 4, pp. 878-897, 2001.

[28] W. Beckner, "Inequalities in fourier analysis," The Annals of Mathematics, vol. 102, no. 1, pp. 159-182, 1975.

[29] A. Dembo, T. M. Cover, and J. A. Thomas, "Information theoretic inequalities," IEEE Transactions on Information Theory, vol. 37, no. 6, pp. 1501-1508.

[30] D. H. Ling, Advanced Mathematics, Tongji University Press, Shanghai, China, 2001.

[31] C. E. Shannon, "A mathematical theory of communication," The Bell System Technical Journal, vol. 27, pp. 379-656, 1948.

[32] T. M. Cover and J. A. Thomas, Elements of Information Theory, John Wiley \& Sons, New York, NY, USA, 2nd edition, 2006. 